\title{
Metabolic characteristics of Rhizoma Coptidis intervention in spontaneously hypertensive rats: Insights gained from metabolomics analysis of serum
}

\author{
WENQING YANG ${ }^{1,2}$, YUE DENG $^{3}$, HONGLEI ZHOU $^{3}$, HAIQIANG JIANG $^{1}$, \\ YUNLUN LI ${ }^{2}$, YANGJUN CHU ${ }^{4}$, XIAOMING WANG ${ }^{3}$ and LILI GONG ${ }^{1}$
}

${ }^{1}$ Experimental Center of Shandong University of Traditional Chinese Medicine, Jinan, Shandong 250355;

${ }^{2}$ Traditional Chinese Medicine Clinical Research Base for Hypertension, Affiliated Hospital of Shandong

University of Traditional Chinese Medicine, Jinan, Shandong 250011; ${ }^{3}$ College of Pharmacy of Shandong

University of Traditional Chinese Medicine, Jinan, Shandong 250355; ${ }^{4}$ The First Clinical Medical College

of Shandong University of Traditional Chinese Medicine, Jinan, Shandong 250355, P.R. China

Received September 2, 2016; Accepted May 18, 2017

DOI: $10.3892 / \mathrm{mmr} .2017 .7119$

\begin{abstract}
The present study aimed to investigate the intervention mechanisms of Rhizoma Coptidis using spontaneously hypertensive rats. A serum metabolomics analysis was performed with high performance liquid chromatography-quadrupole/time of flight mass spectrometer in positive mode. The obtained data were further analyzed by principal component and partial least-squares discriminant analysis to reveal differentiating metabolites. The pattern of metabolites in the serum after Rhizoma Coptidis exhibited distinct alterations. A total of 10 potential biomarkers were significantly altered in the serum and may be associated with the underlying mechanism. Alterations were primarily associated with phospholipid metabolism, fatty acid metabolism, amino acid metabolism and arachidonic acid metabolism. In addition, biochemical alterations in potential biomarkers were associated with inflammation, nitric oxide production, platelet aggregation and endothelial function. By analyzing and verifying the specific biomarkers, metabolomics may be helpful to further understand the underlying therapeutic mechanism of Rhizoma
\end{abstract}

\footnotetext{
Correspondence to: Professor Yunlun Li, Traditional Chinese Medicine Clinical Research Base for Hypertension, Affiliated Hospital of Shandong University of Traditional Chinese Medicine, 42 Wenhua Xi Road, Jinan, Shandong 250011, P.R. China E-mail: yunlun.lee@hotmail.com
}

Dr Haiqiang Jiang, Experimental Center of Shandong University of Traditional Chinese Medicine, 4655 Daxue Road, Jinan, Shandong 250355, P.R. China

E-mail: jhq12723@163.com

Key words: Rhizoma Coptidis, metabolomic, hypertension, high performance liquid chromatography-quadrupole/time of flight mass spectrometer
Coptidis. Metabolomics is a powerful tool used to investigate the therapeutic effects of herbal medicine with multiple targets.

\section{Introduction}

Individuals with high blood pressure are at greater risk of developing coronary heart disease and peripheral artery disease (1). It is a complex, multi-factorial disease, and the underlying physiological and metabolic drivers remain to be fully elucidated. However, patients with hypertension exhibit metabolite alterations.

As an important branch of system biology, metabolomics, developed following genomics and proteomics, deals with a small number of molecules $(<1,000 \mathrm{Da})$ contained within a cell or organism under a given set of conditions. It is a 'discovery' driven holistic evaluation of changes in the expression patterns of hundreds of metabolites, for example, following exposure to environmental stimuli or certain medicines (2). Metabolomics technology has been widely applied in the medical field, and has great potential in the diagnosis of disease and understanding the underlying mechanisms of drugs (3-5). Therefore, performing metabolomic analysis of the metabolic perturbations during hypertension may result in an enhanced understanding of the pathogenesis and effects of drugs.

In the treatment of hypertension, traditional Chinese medicine (TCM) possesses a unique theoretical system and methods of control. Chinese herbal medicines provided a rational means for hypertension. Rhizoma Coptidis, a classical heat-clearing and detoxifying herb, possesses multispectrum therapeutic activities, including antihyperlipidemia, antihypertension, anti-inflammatory and antioxidant effects (6). Rhizoma Coptidis contains a number of isoquinoline alkaloids, in which berberine is the primary component and accounts for $\sim 5-8 \%$. Berberine has a protective effect on high blood pressure and cardiac dysfunction in myocardial hypertrophy model rats induced with L-thyroxine, associated with increased nitric oxide (NO) content (7). Recent studies have indicated that Rhizoma Coptidis serves an important role in treating 
hypertension $(8,9)$. The present study used serum metabolomics based on data generated from high performance liquid chromatography (HPLC)-quadrupole/time of flight (TOF) mass spectrometer (MS), to clarify the underlying therapeutic mechanism of Rhizoma Coptidis treatment in spontaneously hypertensive rats (SHRs).

\section{Materials and methods}

Chemicals and reagents. Acetonitrile, methanol and formic acid of HPLC grade were purchased from Tedia Co., Inc. (Fairfield, OH, USA). All aqueous solutions were prepared using ultrapure Milli-Q water (EMD Millipore, Billerica, MA, USA).

Preparation of Rhizoma Coptidis. Dried Rhizoma Coptidis (a member of the Ranunculaceae family; collected in Mount Emei, Sichuan, China; lot no. 120906) was authenticated on the basis of chemical analysis in accordance with the Chinese Pharmacopoeia (10). The berberine content (pharmacologically active component) was determined by referring to high-performance liquid chromatography-mass spectrometry (unpublished data) and was $7.63 \mathrm{mg} \cdot \mathrm{g}^{-1}$. Other alkaloids included jatrorrhizine hydrochloride $\left(6.01 \mathrm{mg} \cdot \mathrm{g}^{-1}\right)$, epiberberine $\left(6.91 \mathrm{mg} \cdot \mathrm{g}^{-1}\right)$, coptisine $\left(5.09 \mathrm{mg} \cdot \mathrm{g}^{-1}\right)$ and palmatine chloride $\left(7.22 \mathrm{mg} \cdot \mathrm{g}^{-1}\right)$. Powdered Rhizoma Coptidis (100 g) was extracted three times with $95 \%$ ethanol $(1,000 \mathrm{ml})$ under thermal reflux for $1 \mathrm{~h}$. Following filtration, the ethanol extract was combined and concentrated under reduced pressure at $60^{\circ} \mathrm{C}$. The resulting residue was dissolved in and extracted with water three times under the same conditions as with ethanol. Following filtration, water was evaporated at $60^{\circ} \mathrm{C}$. Extracted solutions were combined to obtain a $1.0 \mathrm{~g} \cdot \mathrm{ml}^{-1}$ extract and were stored at $4^{\circ} \mathrm{C}$.

Animal experiment. A total of 12 male SHRs (age, 12 weeks; body weight, 200-250 g) and six male Wistar-Kyoto (WKY) rats (age, 12 weeks; body weight, 230-270 g) were supplied by the Vital River Laboratory Animal Technology Co., Ltd. (Beijing, China), certificate no.: SCXK (Beijing) 2012-0001. All studies were performed according to the guidelines of the National Research Council of China and approved by the Animal Ethics Committee of Affiliated Hospital of Shandong University of Traditional Chinese Medicine (Shandong, China). The 12 SHRs were divided randomly into the model group $(n=6)$ and Rhizoma Coptidis group $(n=6)$ and the 6 WKY were defined as the healthy control group. All rats were kept under standard animal conditions at a regulated temperature $\left(17-25^{\circ} \mathrm{C}\right)$, humidity $(45-60 \%)$ and a 12 -h light/dark cycle. They were allowed free access to food and water throughout the study. The Rhizoma Coptidis group was administered with Rhizoma Coptidis at a dose of $10.001 \mathrm{~g} \cdot \mathrm{kg}^{-1}$. The normal control group and the model group received an equal volume of physiological saline. In accordance with the principle of the same volume and the different concentration, all animals were administered by gastric infusion once daily for 4 continuous weeks.

Blood pressure measurement. Blood pressure was measured via the tail artery prior to and following Rhizoma Coptidis administration at 1, 2, 3 and 4 weeks. Measurement data was expressed as the mean \pm standard error and for multiple group comparisons one-way analysis of variance was used followed by Tukey's test. Data were analyzed using SPSS software (version 17.0; SPSS Inc., Chicago, IL, USA). P<0.05 was considered to indicate a statistically significant difference.

Collection and preparation of serum samples. Serum was collected from the abdominal aorta at day 28 and was immediately centrifuged at $1,780 \mathrm{xg}$ for $15 \mathrm{~min}$ at $4^{\circ} \mathrm{C}$. The supernatant was transferred into a glass sampling vial and stored at $-80^{\circ} \mathrm{C}$. Prior to analysis, $300 \mu 1$ serum was mixed with $600 \mu \mathrm{l}$ acetonitrile and vortexed for $1 \mathrm{~min}$. Following centrifugation at $1,780 \mathrm{xg}$ for $15 \mathrm{~min}$ at $4^{\circ} \mathrm{C}$, the serum sample was filtered through a $0.22-\mu \mathrm{m}$ membrane and transferred to a $1.5-\mathrm{ml}$ glass vial, prior to storage at $-20^{\circ} \mathrm{C}$ until analysis.

HPLC-TOF/MS analysis. HPLC-TOF/MS analysis was performed using an Agilent-1260 LC system coupled with an electrospray ionization source (Agilent Technologies, Inc., Santa Clara, CA, USA) and an Agilent 6230 time-of-flight (TOF) mass spectrometer (Agilent Technologies, Inc.). Chromatographic separations were performed on a $2.1 \times 100 \mathrm{~mm}$ Halo- $\mathrm{C}_{18}$ column $(2.7 \mu \mathrm{m})$ with a binary solvent system (solvent $\mathrm{A}$ : water with $0.1 \%$ formic acid; solvent $\mathrm{B}$ : acetonitrile). The gradient elution program for analysis of serum samples started from $10 \%$ B to $60 \%$ B at $0-15 \mathrm{~min}$, held at $60 \%$ for $7 \mathrm{~min}$, changed to $100 \%$ B over $22-24 \mathrm{~min}$, maintained a strong elution for $10 \mathrm{~min}$, then returned to the initial status and re-equilibrated for $10 \mathrm{~min}$. The flow rate was $0.35 \mathrm{ml} \cdot \mathrm{min}^{-1}$ and the injection volume was $5 \mu \mathrm{l}$. The column was maintained at $25^{\circ} \mathrm{C}$.

Mass detection was operated in positive mode with the following optimized conditions: Capillary voltage, 4,000 V; drying gas flow, $11 \mathrm{l} \cdot \mathrm{min}^{-1}$; gas temperature: $350^{\circ} \mathrm{C}$; nebulizer pressure, 35 psig; fragmentor voltage, $140 \mathrm{~V}$; skimmer voltage, $60 \mathrm{~V}$. Data were collected in centroid mode from 100 to $1,000 \mathrm{~m} / \mathrm{z}$. Samples were analyzed randomly for unbiased measurement with tune mixture solution $(\mathrm{m} / \mathrm{z} 121.050873$ and 922.009798) as internal standards to ensure accuracy and reproducibility.

Data processing and statistical analysis. The raw data were converted to CEF format by Mass Hunter Qualitative Analysis software version B.06.00 (Agilent Technologies, Inc.), imported into Mass Profiler Professional (MPP; Agilent Technologies, Inc.) to continue the preliminary calibration of retention time, abundance and mass range. The data from each sample was then normalized to the summed total area and was inputted into Mass Hunter Qualitative Analysis software a second time to execute regression analysis. Subsequently, data were processed by MPP to continue filter flags and significance analysis. The significance of variation between multiple groups was determined by one-way analysis of variance followed by Tukey's test and the foldchange ( $\geq 2)$. $\mathrm{P}<0.05$ was considered to indicate a statistically significant difference. The resulting three-dimensional matrix, including sample name, peak intensity and peak retention time was introduced into the SIMCA-P software package (version 11.5; Sartorius AB, Göttingen, Germany), 


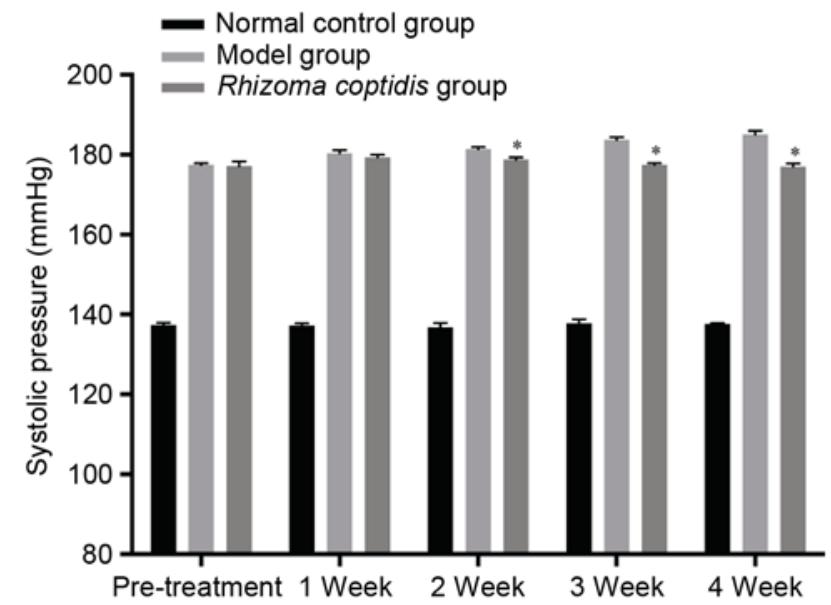

Figure 1. Systolic blood pressure in the normal control, model or Rhizoma Coptidis groups prior to treatment, or following 1,2,3 and 4 weeks of administration. ${ }^{*} \mathrm{P}<0.05$ vs. model group.

where multivariate analysis, including principal component analysis (PCA) and partial least-squares-discriminant analysis (PLS-DA) were performed for pattern recognition. The $\mathrm{R}^{2} \mathrm{Y}$ and $\mathrm{Q}^{2}$ parameters indicated the goodness of fit and the ability of prediction, respectively. Importance for projection (VIP) values produced during PLS-DA was applied to identify a variable that contributed greatly to the classification. Variables with VIP values $>1$ were considered to be statically significant and treated as candidate difference variables (11). In order to further identify these variables, publicly-accessible metabolomics databases were used, including the Human Metabolome Database (www.hmdb.ca), METLIN metabolomics database (metlin.scripps.edu) and the Kyoto
Encyclopedia of Genes and Genomes database (www.kegg. com). In order to explore the metabolic pathways between variables, the MetaboAnalyst 3.0 pathway analysis module (www.metaboanalyst.ca) was used to carry out enrichment analysis and topological analysis of metabolic pathways.

\section{Results and Discussion}

Blood pressure of rats in different groups. Systolic blood pressure (SBP) in the model group and Rhizoma Coptidis group was elevated prior to intervention compared with the healthy control group (Fig. 1). SBP of SHRs treated with Rhizoma Coptidis was decreased compared with the model group $(\mathrm{P}<0.05)$ at 2, 3 and 4 weeks, which reached a minimum at 4 weeks (Fig. 1).

Multivariate data analysis and pattern recognition. Fig. 2 demonstrates examples of HPLC-TOF/MS total ion chromatograms (TIC) of serum samples from each experimental group. PCA was initially used as a starting point for analysis to visualize possible intrinsic clusters and trends among the observations. PCA is additionally used to investigate whether each group is separated and determine metabolic distinction (12). The unsupervised PCA model was used to separate serum samples into three blocks following intervention, which indicated that they had different metabolic profiles (Fig. 3).

PLS-DA, a more sophisticated supervised projection method with the adoption of a UV scale, may be applied to an MS data set (13). In the PLS-DA scores and trajectory analysis of score plots (Fig. 4), the samples from different groups were sorted into different quadrants, with no overlap, which demonstrated that Rhizoma Coptidis effectively altered the

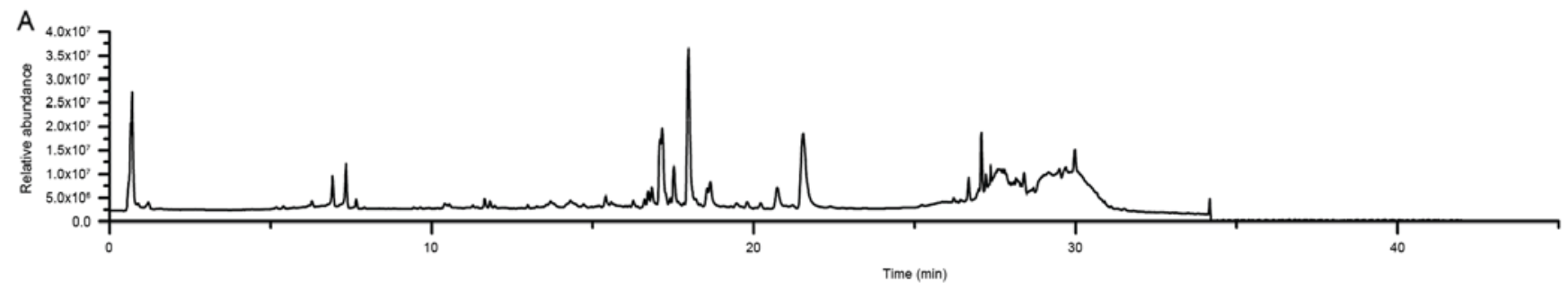

B

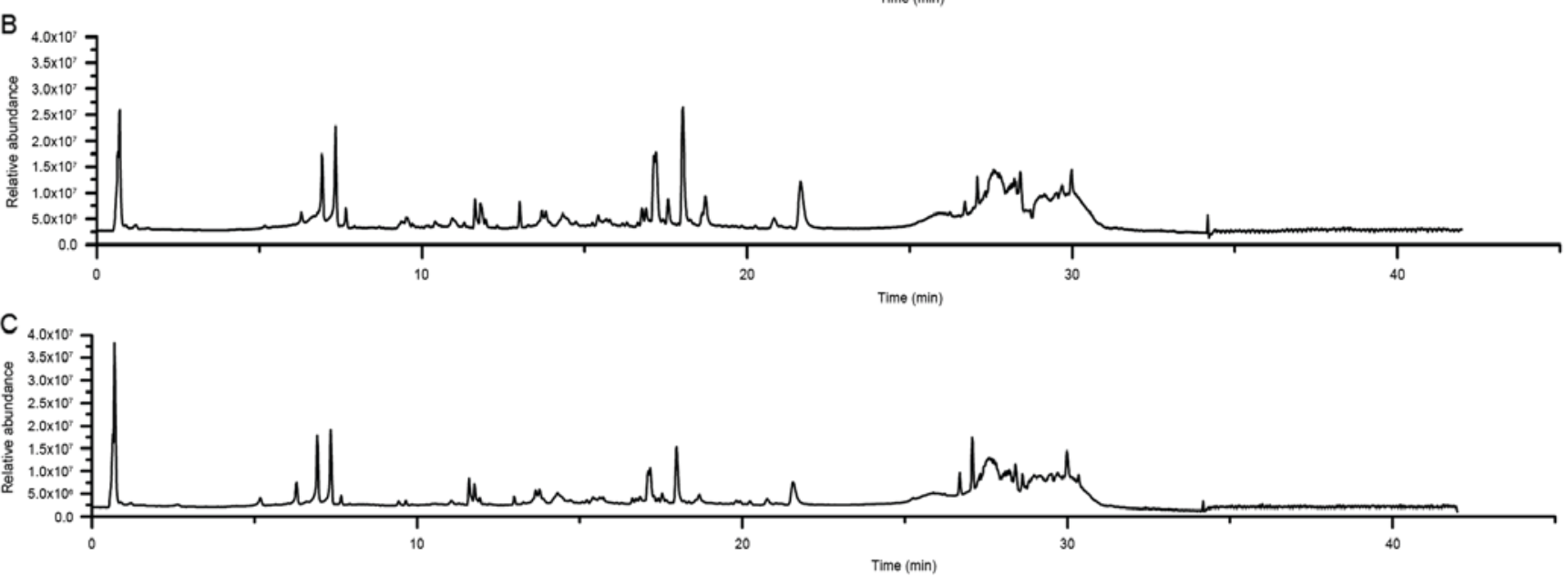

Figure 2. High performance liquid chromatography-quadrupole/time of flight/mass spectrometer total ion chromatograms of serum samples from the (A) normal control group, (B) model group and (C) Rhizoma Coptidis group, in positive mode. 
Table I. Identification of ions and their alteration trend in positive mode.

\begin{tabular}{|c|c|c|c|c|c|c|c|}
\hline \multirow[b]{2}{*}{ No. } & \multirow[b]{2}{*}{$\begin{array}{l}\text { Retention } \\
\text { time } \\
(\mathrm{min})\end{array}$} & \multirow[b]{2}{*}{ Exact mass } & \multirow[b]{2}{*}{ Formula } & \multirow[b]{2}{*}{ Identification } & \multicolumn{2}{|c|}{ Change trend ${ }^{\mathrm{a}}$} & \multirow[b]{2}{*}{ Pathway } \\
\hline & & & & & $\begin{array}{l}\text { Healthy } \\
\text { control } \\
\text { group }\end{array}$ & $\begin{array}{l}\text { Rhizoma } \\
\text { coptidis } \\
\text { group }\end{array}$ & \\
\hline 1 & 0.70 & 220.0848 & $\mathrm{C}_{11} \mathrm{H}_{12} \mathrm{~N}_{2} \mathrm{O}_{3}$ & 5-Hydroxy-L-tryptophan & $\uparrow$ & $\uparrow$ & Tryptophan metabolism \\
\hline 2 & 26.68 & 256.2402 & $\mathrm{C}_{14} \mathrm{H}_{29} \mathrm{COOH}$ & Fatty acid & $\downarrow$ & $\downarrow$ & Glycerolipid metabolism \\
\hline 3 & 28.82 & 296.3079 & $\mathrm{C}_{20} \mathrm{H}_{40} \mathrm{O}$ & Thromboxane & $\downarrow$ & $\downarrow$ & Arachidonic acid metabolism \\
\hline 4 & 27.08 & 284.2715 & $\mathrm{C}_{9} \mathrm{H}_{13} \mathrm{~N}_{2} \mathrm{O}_{9} \mathrm{P}$ & Stearic acid & $\downarrow$ & $\downarrow$ & Biosynthesis of unsaturated fatty acids \\
\hline 5 & 32.19 & 757.5622 & $\mathrm{C}_{42} \mathrm{H}_{80} \mathrm{NO}_{8} \mathrm{P}$ & PC (18:2(9Z,12Z)/16:0) & $\downarrow$ & $\downarrow$ & Glycerophospholipid metabolism \\
\hline 6 & 27.21 & 332.2715 & $\mathrm{C}_{22} \mathrm{H}_{36} \mathrm{O}_{2}$ & Adrenic acid & $\downarrow$ & $\downarrow$ & Biosynthesis of unsaturated fatty acids \\
\hline 7 & 33.13 & 702.5676 & $\mathrm{C}_{39} \mathrm{H}_{79} \mathrm{~N}_{2} \mathrm{O}_{6} \mathrm{P}$ & SM (d18:0/16:1(9Z)) & $\uparrow$ & $\uparrow$ & Sphingolipid metabolism \\
\hline 8 & 15.67 & 330.2559 & $\mathrm{C}_{22} \mathrm{H}_{34} \mathrm{O}_{2}$ & Docosapentaenoic acid & $\uparrow$ & $\uparrow$ & Biosynthesis of unsaturated fatty acids \\
\hline 9 & 28.67 & 715.5516 & $\mathrm{C}_{39} \mathrm{H}_{74} \mathrm{NO}_{8} \mathrm{P}$ & PE (18:1(9Z)/16:1(9Z)) & $\downarrow$ & $\downarrow$ & Glycerophospholipid metabolism \\
\hline 10 & 19.36 & 509.3481 & $\mathrm{C}_{25} \mathrm{H}_{52} \mathrm{NO}_{7} \mathrm{P}$ & LysoPC (17:0) & $\uparrow$ & $\uparrow$ & Glycerophospholipid metabolism \\
\hline
\end{tabular}

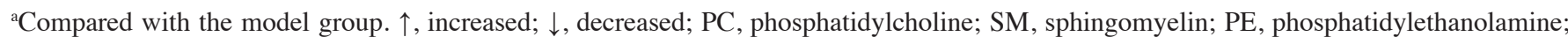
LysoPC, lysophospholipids.

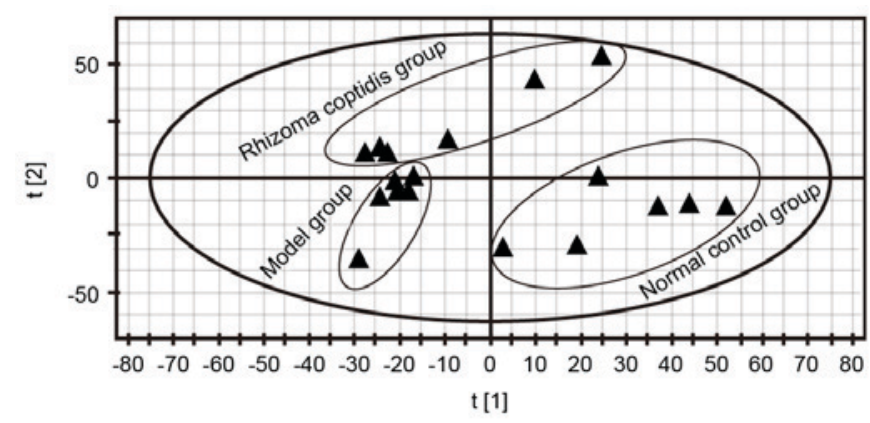

Figure 3. The principal component analysis plot of serum data from the normal control group, model group and Rhizoma Coptidis group ( $\mathrm{n}=6 / \mathrm{group})$. $\mathrm{t}[1]$, first principle component score; $\mathrm{t}[2]$, second principle component score.

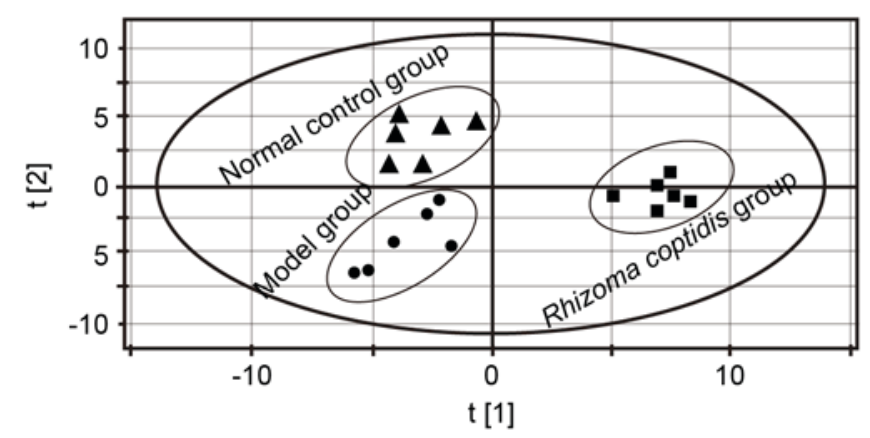

Figure 4. The partial least-squares-discriminant analysis plot of serum data from the normal control group, model group and Rhizoma Coptidis group $(n=6 /$ group). $t[1]$, first principle component score; $t[2]$, second principle component score.

physiological and metabolic conditions of rats. $\mathrm{R}^{2} \mathrm{Y}$ and $\mathrm{Q}^{2}$ of the PLS-DA model were $0.856,0.793$ and 0.841 , respectively. The result indicated that a well-fitted PLS-DA model was established.

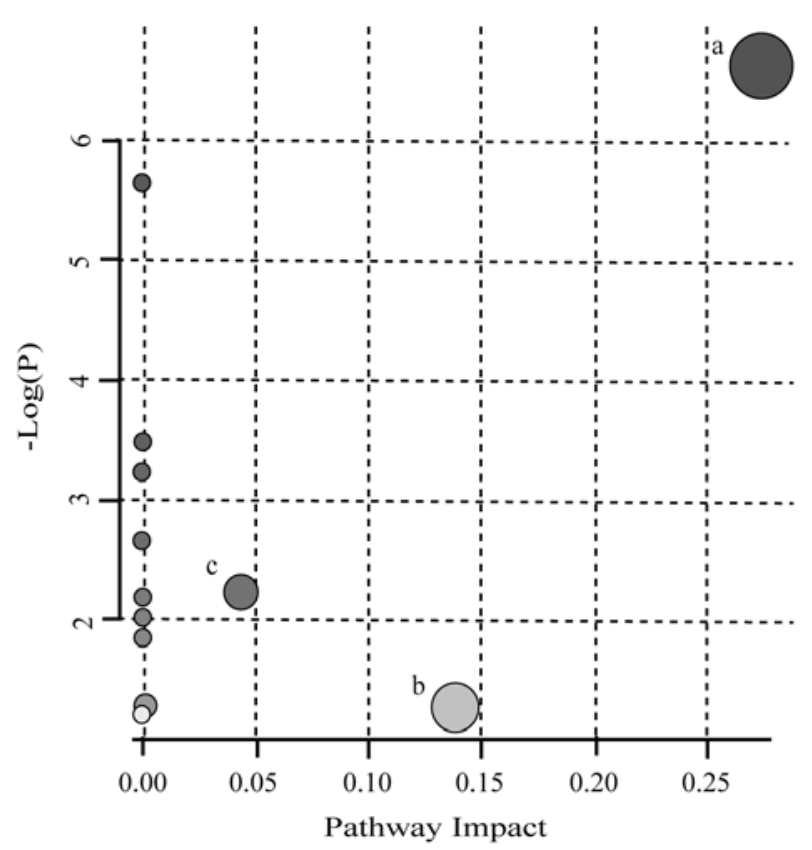

Figure 5. Summary of pathway analysis using the pathway analysis module The color and size of each circle are determined by the P-value of the metabolic pathway and the importance of the pathway. a, glycerophospholipid metabolism; b, tryptophan metabolism; c, glycosylphosphatidylinositol-anchor biosynthesis.

Identification of potential biomarkers. After the processing of PCA and PLS-DA, metabolites that significantly contributed to the clustering were identified according to a threshold of VIP. Ions with VIP values $>1$ demonstrated potential endogenous components. Ultimately, 10 potential biomarkers were identified. Compared with the model group, serum levels of fatty acid, stearic acid, thromboxane (TX), phosphatidylcholine (PC) [18:2 (9Z, 12Z)/16:0], adrenic acid and phosphatidylethanolamine (PE) [18:1 (9Z)/16:1 (9Z)] were increased following 


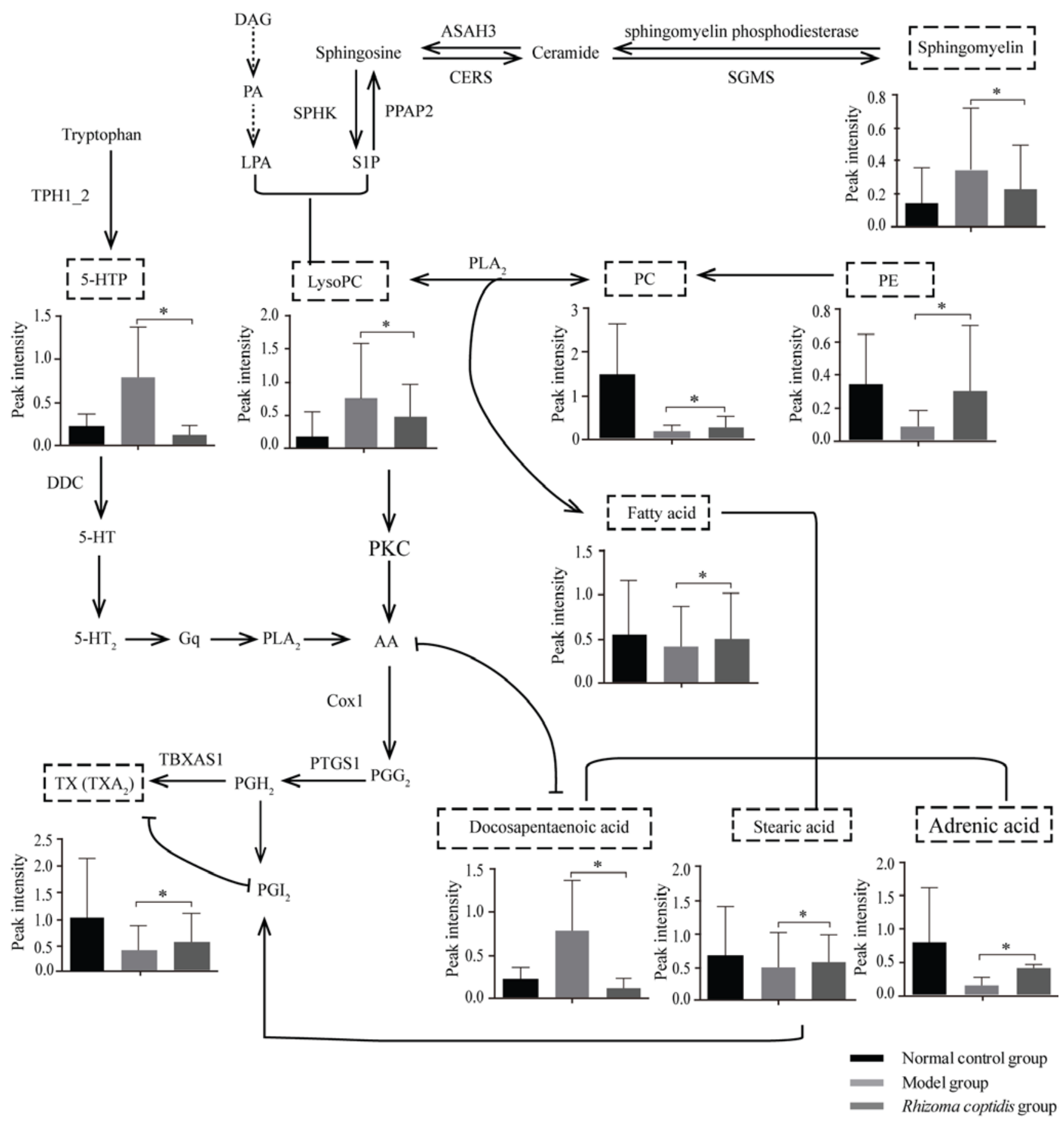

Figure 6. Metabolic network of the altered biomarkers in the normal control group, model group and Rhizoma Coptidis group. P-values were calculated using analysis of variance. $n=6 /$ group. ${ }^{*} \mathrm{P}<0.05$. Dashed line boxes represent potential biomarkers. Straight arrows represent the association between metabolites. Dashed arrows represent indirect reaction generation. TPH1_2, tryptophan 5-monooxygenase; 5-HTP, 5-hydroxy-1-tryptophan; DDC, aromatic-1-amino-acid decarboxylase; 5-HT, 5-hydroxytryptamine; PLA, lysophosphatidic acid; PA, phosphatidic acid; DAG, diacylglycerol; S1P, sphingosine 1 phosphate; SPHK, sphingosine kinase; PPAP2, phosphatidate phosphatase; ASAH3, alkaline ceramidase; CERS, ceramide synthetase; SGMS, sphingomyelin synthase; LysoPC, lysophospholipids; PLA2, phospholipase A2; AA, arachidonic acid; COX1, cyclooxygenase 1; PTGS1, prostaglandin-endoperoxide synthase 1; PGG2, prostaglandin G2; PGH2, prostaglandin H2; TBXAS1, thromboxane-A synthase; PGI2, prostaeyelin; TX, thromboxane; TXA 2 , thromboxane A2; PE, phosphatidylethanolamine; PC, phosphatidylcholine; Gq, guanine nucleotide-binding protein G(q) subunit $\alpha$.

intervention, while 5-hydroxy-L-tryptophan (5-HTP), sphingomyelin [d18:0/16:1 (9Z)], docosapentaenoic acid and LysoPC (17:0) were decreased. The differences and alteration trend of these biomarkers are summarized in Table I.

Metabolic pathway analysis and biological function analysis. The 10 identified biomarkers were imported into the pathway analysis module to explore the possible signaling pathways that were affected by Rhizoma Coptidis. The metabolic pathway analysis with the pathway analysis module revealed that glycerophospholipid metabolism, tryptophan metabolism and glycosylphosphatidylinositol anchor biosynthesis were identified to be important metabolic pathways (Fig. 5). Based on the knowledge of these biomarkers and online databases, 
a network of Rhizoma Coptidis intervention-associated metabolic pathways was generated (Fig. 6), indicating that the antihypertensive effect of Rhizoma Coptidis is associated with the certain endogenous metabolites.

Phospholipid metabolism disorders and inflammation. There is widespread evidence that altered lipid metabolism is involved in the pathogenesis of hypertension $(14,15)$. Numerous studies have demonstrated that abnormal phospholipid metabolism serves an essential role in the development of intimal injury and vascular disease $(16,17)$. Traditionally, total serum cholesterol, triglyceride, high-density lipoprotein (HDL) and low-density lipoprotein (LDL) concentrations are considered important and clinically useful biomarkers of hypertension $(18,19)$. With the rapid development of metabolomics, the identification of potential lipid biomarker signatures may provide clues for the investigation of the pathogenesis of hypertension. Kulkarni et al (20) demonstrated through plasma lipidomic profiling that a disturbance in diacylglycerol metabolism is an independent biomarker of hypertension. In the present study, four different phospholipids species were identified: Lysophospholipids (LysoPC), PE, PC and sphingomyelin (SM). The results of the present study revealed that PC [18:2 (9Z, 12Z)/16:0] and PE [18:1 (9Z)/16:1 (9Z)] were significantly decreased in the model group compared with the healthy control group, whereas sphingomyelin [d18:0/16:1 (9Z)] and LysoPC (17:0) were significantly increased. There is a tendency to return to normal following intervention by Rhizoma Coptidis. The results suggested that Rhizoma Coptidis may correct phospholipid metabolism disorder.

LysoPC is a simple phospholipid signaling molecule. Lysophosphatidic acid (LPA) and sphingosine 1 phosphate (S1P) are two primary bioactive lysophospholipids. They may couple with multiple types of $\mathrm{G}$ proteins to activate a range of downstream effectors. LPA and S1P regulate endothelial functions, and serve a role in the regulation of angiogenesis and blood vessel integrity (21-23). LysoPC additionally promotes the development of inflammation. LysoPC induces the activation of phospholipase A2 via activation of protein kinase $\mathrm{C}$, and then induces the hydrolysis of membrane phospholipids and release of free arachidonic acid (24). The proportion of $\mathrm{PE}$ and $\mathrm{PC}$ combined is $75 \%$ in glycerol phospholipid composition. In the lipid metabolism network, PC is the primary source of arachidonic acid that is a precursor of eicosanoids, which serve an important role in inflammatory processes. During inflammation, $\mathrm{PC}$ is converted to arachidonic acid. PE is an instantaneous source and may be quickly added. When inflammation subsides, $\mathrm{PC}$ is converted to arachidonic acid at a slower pace. SM is the only sphingolipid class that additionally belongs to the phospholipid class (25). The biologically active sphingomyelin metabolites (SMM), sphingosine-1-phosphate (S1P), sphingosylphosphorylcholine, sphingosine and ceramide are important lipid mediators, which are generated upon cell activation from membrane phospholipids as part of the sphingomyelin cycle (26). S1P is the most potent intercellular signaling molecule in the sphingolipid family. S1P serves vital roles in angiogenesis, endothelial cell proliferation and migration and vascular permeability (27). Yogi et al (28) identified a novel signaling pathway linking S1P/S1P1 receptors to specific proinflammatory signaling pathways via epidermal growth factor receptor and platelet-derived growth factor transactivation. Such molecular events may contribute to vascular inflammation in hypertension. The alteration in LysoPC, PE, PC and SM by this test demonstrated that inflammatory reactions exist in hypertension (29), and suggest that Rhizoma Coptidis may be an anti-inflammatory drug $(6,30)$.

Fatty acid metabolism and endothelial NO. In the model group, fatty acids and stearic acid were significantly increased and docosapentaenoic acid was decreased compared with the healthy group. This suggested that the aberrant distribution of fatty acids contributes to the pathogenesis of hypertension (31). Stearic acid is a useful type of saturated fatty acid. Docosapentaenoic acid is an essential omega-3 fatty acid. An important function of docosapentaenoic acid is the transport and oxidation of cholesterol. It may reduce plasma cholesterol, triglycerides and LDL, while increasing HDL. In addition, docosapentaenoic acid and arachidonic acid (AA) have the same metabolic pathway, and are the common substrate of a number of metabolic enzymes. When they simultaneously exist, they have a competing inhibition effect. Therefore, docosapentaenoic acid may inhibit the cyclooxygenase metabolic of AA to some extent, thereby reducing inflammation derivatives, including TXA2 and prostaeyelin $\left(\mathrm{PGI}_{2}\right)$. The fatty acids in serum may cause vasoconstriction and blood pressure elevation by increasing the concentration of blood $\mathrm{Ca}^{2+}$ or exciting the sympathetic nervous system $(32,33)$. Fatty acids may locate to cell membrane phospholipids as the second messengers to alter membrane function, directly affect vascular endothelial cells and endothelial NO synthesis, which may reduce the content or the activity of endogenous NO (34). Table I demonstrates that Rhizoma Coptidis successfully reversed the fatty acid metabolism disorder. The results suggested that Rhizoma Coptidis may reduce blood pressure by lowering serum fatty acids content and may improve the activity of endothelial NO.

TX, adrenic acid and arachidonic acid metabolism. TX is produced in platelets by TX synthase, which is produced from endoperoxides by the cyclooxygenase (COX) enzyme from AA. TX is a vasoconstrictor, potent hypertensive agent and facilitates the clumping of platelets. The two primary TXs are $\mathrm{TXA}_{2}$ and $\mathrm{TXB}_{2}$. Adrenic acid is metabolized by COX, lipoxygenases and cytochrome $\mathrm{P} 450 \mathrm{~s}$ to dihomo-eicosanoids (35). Under physiological conditions, $\mathrm{TXA}_{2}$ and $\mathrm{PGI}_{2}$ are in a state of dynamic equilibrium. $\mathrm{PGI}_{2}$ is released and synthesized by endothelial cells. It is a biologically active substance, which has vasodilator and anti-platelet aggregation properties. $\mathrm{TXA}_{2}$ and $\mathrm{PGI}_{2}$ imbalance is one of the causes of platelet aggregation, vascular spasm or thrombosis. In the pathologic process of hypertension, high blood pressure damages the endothelium, then collagen and fibers under the skin are exposed to $\mathrm{TXA}_{2}$ released by platelets (36). Subsequently, $\mathrm{TXA}_{2}$ may allow blood pressure to continually rise via the inhibition of $\mathrm{PGI}_{2}$ synthesis, impact on the vascular permeability and promote the proliferation of smooth muscle cells. However, adrenic acid metabolites serve a potential role in the regulation of vasodilation. Adrenic acid is metabolized in human vascular endothelial cells to $\mathrm{PGI}_{2}$, which inhibited thrombin-induced platelet aggregation (37). The present study revealed that in the 
model group, the alteration in TX and adrenic acid suggested that there is an association between hypertension, and TX and adrenic acid. Following intervention with Rhizoma Coptidis, TX and adrenal acid metabolism were improved. This suggested that Rhizoma Coptidis may be involved in anti-platelet aggregation.

5-HTP and the sympathetic nervous system. 5-HTP is an aromatic amino acid naturally produced by the body from the essential amino acid L-tryptophan. It may affect mood, sleep and appetite. 5-HTP has been used clinically for $>30$ years, and has been demonstrated to be effective in treating a wide variety of conditions, including depression, chronic headache and insomnia (38-40). In animal and human experiments, 5-HTP promoted the metabolism of catecholamines, in particular to promote dopamine and norepinephrine release (41). These amino acids therefore affect the activity of the sympathetic nervous system. Increased sympathetic activity is a primary cause of hypertension. 5-HTP is the immediate precursor of the neurotransmitter 5-hydroxytryptamine (5-HT) and serves a significant role in the 5-HT pathway. L-tryptophan is hydroxylated by tryptophan 5-hydroxylase to form 5-HTP, following which aromatic amino acid decarboxylases catalyze the formation of 5-HT (42). 5-HT has multiple vascular effects, depending on the different 5-HT receptor subtypes (43). $5-\mathrm{HT}_{1} \mathrm{R}$ mediates vasodilating activity and $5-\mathrm{HT}_{2} \mathrm{R}$ mediates vasoconstriction $(44,45)$. In the present study, the serum levels of 5-HTP in the Rhizoma Coptidis group reduced compared with the model group. This suggested that Rhizoma Coptidis regulates metabolic pathways of catecholamine and 5-HT to reduce blood pressure. However, the specific underlying mechanism of action of Rhizoma Coptidis on 5-HTP requires further investigation.

In conclusion, clinical practice has demonstrated that decreasing blood pressure cannot completely protect and reverse damaged organs or tissue caused by high blood pressure. The primary reason is multiple risk factors in patients with hypertension interact and influence each other. Therefore, treatment of hypertension requires drugs that target multiple targets and metabolic pathways. In the present study, by applying metabolomics technology, the therapeutic efficacies and mechanism of Rhizoma Coptidis on SHRs was investigated.

There were metabolic differences between the model group and Rhizoma Coptidis groups, and Rhizoma Coptidis reversed the levels of 10 perturbed metabolites. These alterations suggested that the antihypertensive effect of Rhizoma Coptidis may involve the regulation of phospholipids, fatty acids, amino acid and arachidonic acid metabolism. However, there were limitations of the research, and the results require further validation. This study provides insights into the underlying therapeutic mechanism of Rhizoma Coptidis for hypertension.

\section{Acknowledgements}

The present study was funded by the National Youth Foundation of China (grant no. 81102549), the National Natural Science Foundation of China (grant no. 81473653) and the Project of Traditional Chinese Medicine Antiviral Collaborative Innovation Center in Shandong Province (grant no. XTCX2014C01-03).

\section{References}

1. Mozaffarian D, Benjamin EJ, Go AS, Arnett DK, Blaha MJ, Cushman M, de Ferranti S, Després JP, Fullerton HJ, Howard VJ, et al: Heart disease and stroke statistics-2015 update: A report from the American heart association. Circulation 131: e29-e22, 2015.

2. Nicholson JK, Connelly J, Lindon JC and Holmes E: Metabonomics: A platform for studying drug toxicity and gene function. Nat Rev Drug Discov 1: 153-161, 2002.

3. Delles C and Husi H: Systems biology approach in hypertension research. Methods Mol Biol 1527: 69-79, 2017.

4. Xu T, Li S, Sun Y, Pi Z, Liu S, Song F and Liu Z: Systematically characterize the absorbed effective substances of Wutou Decoction and their metabolic pathways in rat plasma using UHPLC-Q-TOF-MS combined with a target network pharmacological analysis. J Pharmaceut Biomed Analy 141: 95-107, 2017.

5. Matsutomo T, Ushijima M, Kodera Y, Nakamoto M, Takashima M, Morihara N and Tamura K: Metabolomic study on the antihypertensive effect of S-1-propenylcysteine in spontaneously hypertensive rats using liquid chromatography coupled with quadrupole-Orbitrap mass spectrometry. J Chromatogr B Analyt Technol Biomed Life Sci 1046: 147-155, 2017.

6. Pang B, Yu XT, Zhou Q, Zhao TY, Wang H, Gu CJ and Tong XL: Effect of Rhizoma coptidis (Huang Lian) on treating diabetes Mellitus. Evid Based Complement Alternat Med 2015: 921416, 2015.

7. Zhao HP, Hong Y, Xie JD, Xie XR, Wang J and Fan JB: Effect of berberine on left ventricular remodeling in renovascular hypertensive rats. Yao Xue Xue Bao 42: 336-341, 2007 (In Chinese).

8. Lan J, Zhao Y, Dong F, Yan Z, Zheng W, Fan J and Sun G: Meta-analysis of the effect and safety of berberine in the treatment of type 2 diabetes mellitus, hyperlipemia and hypertension. J Ethnopharmacol 161: 69-81,2015.

9. Liu L, Liu J, Huang Z, Yu X, Zhang X, Dou D and Huang Y: Berberine improves endothelial function by inhibiting endoplasmic reticulum stress in the carotid arteries of spontaneously hypertensive rats. Biochem Biophys Res Commun 458: 796-801, 2015.

10. China Pharmacopoeia Commission: Pharmacopoeia of the People's Republic of China. Beijing China Medical Science Press, 2015

11. Chong IG and Jun CH: Performance of some variable selection methods when multicollinearity is present. Chemom Intell Lab Sys 78: 103-112, 2005

12. Wang TJ, Larson MG, Vasan RS, Cheng S, Rhee EP, McCabe E, Lewis GD, Fox CS, Jacques PF, Fernandez C, et al: Metabolite profiles and the risk of developing diabetes. Nat Med 17: 448-453, 2011.

13. Suzuki S, Sumi K and Matsubara M: Cardiac autonomic control immediately after exercise in female distance runners. J Physiol Anthropol 27: 325-332, 2008

14. Kistler T and Weisser B: Correlation between disorders of lipid metabolism and hypertension in 10,892 participants in the Heureka Study. Schweiz Rundsch Med Prax 82: 1222-1233, 1993.

15. Lopes FH, Silva HB, Soares JA, Filho B, Consolim-Colombo FM, Giorgi DM and Krieger EM: Lipid metabolism alterations in normotensive subjects with positive family history of hypertension. Hypertension 30: 629-631, 1997.

16. Kolliputi N, Galam L, Parthasarathy PT, Tipparaju SM and Lockey RF: NALP-3 inflammasome silencing attenuates ceramide-induced transepithelial permeability. J Cell Physiol 227: 3310-3316, 2012.

17. Jernigan PL, Makley AT, Hoehn RS, Edwards MJ and Pritts TA: The role of sphingolipids in endothelial barrier function. Biol Chem 396: 681-691, 2015.

18. Halperin RO, Sesso HD, Ma J, Buring JE, Stampfer MJ and Gaziano JM: Dyslipidemia and the risk of incident hypertension in men. Hypertension 47: 45-50, 2006.

19. Freitas MP, Loyola Filho AI and Lima-costa MF: Dyslipidemia and the risk of incident hypertension in a population of community-dwelling Brazilian elderly: The bambui cohort study of aging. Cad Saúde Pública 27: S351-S359, 2011.

20. Kulkarni H, Meikle PJ, Mamtani M, Weir JM, Barlow CK, Jowett JB, Bellis C, Dyer TD, Johnson MP, Rainwater DL, et al: Plasma lipidomic profile signature of hypertension in Mexican American families: specific role of diacylglycerols. Hypertension 62: 621-626, 2013.

21. Lee H, Goetzl EJ and An S: Lysophosphatidic acid and sphingosine 1-phosphate stimulate endothelial cell wound healing. Am J Physiol Cell Physiol 278: C612-C618, 2000. 
22. Lee MJ, Thangada S, Claffey KP, Ancellin N, Liu CH, Kluk M, Sha'afi RI, Volpi M and Hla T: Vascular endothelial cell adherens junction assembly and morphogenesis induced by sphingosine-1-phosphate.Cell 99: 301-312, 1999.

23. Lee H, Lin CI, Liao JJ, Lee YW, Yang HY, Lee CY, Hsu HY and $\mathrm{Wu}$ HL: Lysophospholipids increase ICAM-1 expression in HUVEC through a Gi- and NF-kappaB-dependent mechanism. Am J Physiol Cell Physiol 287: C1657-C1666, 2004.

24. Yang HH, Qin F, Liang QL, Wang Y, Wang YM and Luo GA: LapRLSR for NIR spectral modeling and its application to online monitoring of the column separation of Salvianolate. Chi Chemical Lett 18: 852-856, 2007.

25. Zhang T, Chen S, Liang X and Zhang H: Development of a mass-spectrometry-based lipidomics platform for the profiling of phospholipids and sphingolipids in brain tissues. Anal Bioanal Chem 407: 6543-6555, 2015.

26. Kolesnick R: The therapeutic potential of modulating the ceramide/sphingomyelin pathway. J Clin Invest 110: 3-8, 2002.

27. Jernigan PL, Makley AT, Hoehn RS, Edwards MJ and Pritts TA The role of sphingolipids in endothelial barrier function. Biol Chem 396: 681-691, 2015.

28. Yogi A, Callera GE, Aranha AB, Antunes TT, Graham D, McBride M, Dominiczak A and Touyz RM: Sphingosine1-phosphate-induced inflammation involves receptor tyrosine kinase transactivation in vascular cells: Upregulation in hypertension. Hypertension 57: 809-818, 2011.

29. Vardas $P$ and Marketou M: CRP in non-dippers: New perspectives and old queries. J Hum Hypertens 22: 447-449, 2008.

30. Wang N, Tan HY, Li L, Yuen MF and Feng Y: Berberine and Coptidis Rhizoma as potential anticancer agents: Recent updates and future perspectives. J Ethnopharmacol 176: 35-48, 2015.

31. Hall WL: Dietary saturated and unsaturated fats as determinants of blood pressure and vascular function. Nutr Res Rev 22: 18-38, 2009.

32. Wilde DW, Massey KD, Walker GK, Vollmer A and Grekin RJ: High-fat diet elevates blood pressure and cerebrovascular muscle $\mathrm{Ca}(2+)$ current. Hypertension 35: 832-837, 2000.

33. Antic V, Dulloo A and Montani JP: Multiple mechanisms involved in obesity-induced hypertension. Heart Lung Circ 12: 84-93, 2003.
34. Grimsgaard S, Bonaa KH, Jacobsen BK and Bjerve KS: Plasma saturated and linoleic fatty acids are independently associated with blood pressure. Hypertension 34: 478-483, 1999.

35. Kopf PG, Zhang DX, Gauthier KM, Nithipatikom K, Yi XY, Falck JR and Campbell WB: Adrenic acid metabolites as endogenous endothelium-derived and zona glomerulosa-derived hyperpolarizing factors. Hypertension 55: 547-554, 2010.

36. Jagroop IA, Clatworthy I, Lewin J and Mikhailidis DP: Shape change in human platelets: Measurement with a channelyzer and visualisation by electron microscopy. Platelets 11: 28-32, 2000.

37. Campbell WB, Falck JR, Okita JR, Johnson AR and Callahan KS: Synthesis of dihomoprostaglandins from adrenic acid $(7,10,13,16$-docosatetraenoic acid) by human endothelial cells. Biochim Biophys Acta 837: 67-76, 1985.

38. van Praag H and de Hann S: Depression vulnerability and 5-hydroxytryptophan prophylaxis. Psychiatry Res 3: 75-83, 1980.

39. Ribeiro CA: L-5-Hydroxytryptophan in the prophylaxis of chronic tension-type headache: A double-blind, randomized, placebo-controlled study. For the Portuguese head society. Headache 40: 451-456, 2000.

40. Guilleminault C, Cathala JP and Castaigne P: Effects of 5-hydroxytryptophan on sleep of a patient with a brain-stem lesion. Electroencephalogr Clin Neurophysiol 34: 177-184, 1973.

41. Li JD, Liu SH and Tang M: Recent progress on study of 5-hydroxytryptophan. Fine Specialty Chemicals 22: 36-39, 2014.

42. Hara R and Kino K: Enhanced synthesis of 5-hydroxy-1-tryptophan through tetrahydropterin regeneration. AMB Express 3: 70, 2013.

43. Bevan J and Heptinsatll S: Serotonin indueed platelet aggregation in whole blood and the effects of ketanserin and mepyramine. Thromb Res 38: 189-194, 1985.

44. MacLean MR, Sweeney G, Baird M, McCulloch KM, Houslay M and Morecroft I: 5-Hydroxytryptamine receptors mediating vasoconstriction in pulmonary arteries from control and pulmonary hypertensive rats. Br J Pharmacol 119: 917-930, 1996.

45. Morecroft I and Maclean MR: 5-hydroxytryptamine receptors mediating vasoconstriction and vasodilation in perinatal and adult rabbit small pulmonary arteries. Br J Pharmacol 125: 69-78, 1998. 